\title{
Controlled Release of Matrix Metalloproteinase-1 Plasmid DNA Prevents Left Ventricular Remodeling in Chronic Myocardial Infarction of Rats
}

\author{
Xue Lin, MD*,†; Hikari Jo, MD**; Takahiro M Ishii, $\mathrm{PhD}^{\dagger}$; Masatoshi Fujita, MD ${ }^{\dagger+}$; \\ Michael Fu, MD*; Keiichi Tambara, MD*; Masaya Yamamoto, PhD ${ }^{*}$; \\ Yasuhiko Tabata, PhD $\$$; Masashi Komeda, MD*, ; Satoshi Matsuoka, MD**
}

\begin{abstract}
Background: The present study investigated whether administration of controlled release matrix metalloproteinase-1 (MMP-1) plasmid DNA prevents left ventricular (LV) remodeling in a rat chronic myocardial infarction (MI) model.

Methods and Results: Rats with a moderate-sized MI were randomized to 2 groups: injection of phosphate buffered saline (PBS) containing microspheres into the peri-infarct area (MI group, $n=14$ ) and injection of cationized gelatin microspheres incorporating MMP-1 plasmid DNA (MI+MMP-1 group, 50 $\mu \mathrm{g}$ MMP-1/20 $\mu \mathrm{l}$; n=14). As a control group $(\mathrm{n}=14)$, rats received neither the coronary artery ligation nor the injection of PBS. Echocardiography, cardiac catheterization and histological studies were performed. At 2 and 4 weeks after the treatment, the MI+MMP-1 group had smaller LV end-diastolic and end-systolic dimensions, better fractional area change and smaller akinetic areas than the MI group. The LV end-systolic elastance and time constant of isovolumic relaxation were also better in the MI+MMP-1 group compared with the MI group 4 weeks after the treatment. Fibrosis evaluated with Masson's trichrome staining was less in the MI+MMP-1 group than the MI group.

Conclusions: Gelatin microspheres for the controlled release of MMP-1 plasmid DNA are promising for improving cardiac remodeling and function when they are administered during the chronic phase of MI. (Circ J 2009;
\end{abstract} 73: $2315-2321$ )

Key Words: Current (INCX) of Na/Ca exchange (NCX); Matrix metalloproteinase-1; Microspheres; Myocardial infarction; Single cardiomyocyte shortening

$\mathbf{S}$ urvivors of myocardial infarction (MI) are at high risk of developing chronic heart failure. ${ }^{1}$ Rapid recanalization of the occluded coronary artery is presently the best clinical treatment for acute MI, which, if carried out in time, enables considerable salvage of the ischemic myocardial cells. Unfortunately, not all patients have the chance for or respond to timely coronary reperfusion therapy because it must be performed within a few hours after the onset of infarction. ${ }^{2}$ Indeed, patients with post-MI heart failure account for nearly half of the candidates for cardiac transplantation. ${ }^{3}$ Consequently, development of an effective therapy for post-MI heart failure is one of the most important issues.

Matrix metalloproteinases (MMPs) are a family of proteolytic enzymes that denature and degrade collagens and other components of the extracellular matrix (ECM). MMPs are involved in many physiological processes, such as embryonic development, ovulation, bone remodeling and wound healing. ${ }^{4}$ Their enhanced activities have also been implicated in numerous disease processes, including arthritis, tumor cell metastasis, periodontal disease, atherosclerosis and MI. ${ }^{5-7}$ In the progression of MI, MMPs are known to play an important role in several aspects of infarct healing, such as early ECM degradation, cell migration (inflammatory cells, fibroblasts), angiogenesis, remodeling of newly synthesized connective tissue and the regulation of growth factor activities. ${ }^{8,9}$ As a superfamily, MMPs consist of at least 16 members, including the collagenase group, gelatinase group and several membrane-type MMPs. The colleganase group consists of MMP-1, MMP-8 and MMP-13. But only MMP-1 is an interstitial colleganase and it degrades the structural collagens of type I, II and III with the characteristic cleavage site for type I collagen. ${ }^{10}$ The early rise of MMP-1 synthesis has been reported in the infarcted myocardium..$^{9}$ The subsequent inactivation of MMP-1 synthesis in the infarcted myocardium leads to collagen accumulation, ${ }^{11}$ and excessive accumulation may result in a stiff and non-compliant left ventricle (LV) and gradual heart dysfunction. Recently, Aoyama et al reported that local delivery of the MMP-1 gene prevents the onset of renal fibrosis in

Received May 28, 2009; revised manuscript received August 7, 2009; accepted August 26, 2009; released online October 2, 2009

*Department of Cardiovascular Surgery, **Department of Physiology and Biophysics, ${ }^{\dagger}$ Department of Physiology, Neurobiology, ${ }^{\dagger} H u m a n$ Health Sciences, Graduate School of Medicine, Kyoto University, Kyoto, Japan, ‡Department of Cardiovascular Research, Sahlgrenska University Hospital/Sahlgrenska, University of Göteborg, Göteborg, Sweden, \$Department of Biomaterials, Field of Tissue Engineering, Institute for Frontier Medical Sciences, Kyoto University, Kyoto and §Department of Cardiovascular Surgery, Nagoya Heart Center, Nagoya, Japan

Mailing address: Xue Lin, MD, Human Health Sciences, Graduate School of Medicine, Kyoto University, 53 Kawara-cho, Shogoin, Sakyo-ku, Kyoto 606-8507, Japan. E-mail: xuelin@kuhp.kyoto-u.ac.jp

All rights are reserved to the Japanese Circulation Society. For permissions, please e-mail: cj@j-circ.or.jp 
streptozotocin-induced diabetic mice. ${ }^{12}$ We hypothesized that local delivery of MMP-1 decreases fibrosis in chronic post-MI hearts and improves cardiac function. The aim of the present study was to determine whether local controlled release of MMP-1 plasmid DNA prevents the progression of myocardial fibrosis and improves cardiac function in rats with chronic MI heart failure.

\section{Methods}

All experimental procedures were carried out in accordance with the guidelines for Animal Experiments in Kyoto University, Japan, and conformed with the "Care and Use of Laboratory Animals" published by the US National Institutes of Health.

\section{Preparation of Cationized Gelatin}

Gelatin samples with an isoelectric point of 9.0 (MW $100,000)$, prepared with pig skin using an acid process, were kindly supplied by Nitta Gelatin Inc, Osaka, Japan. The carboxyl groups of gelatin were chemically converted by introducing amino groups for cationization of gelatin. ${ }^{13,14}$ Briefly, ethylenediamine (Wako Pure Chemical, Ltd, Osaka, Japan) and 1-ethyl-3-(3-dimethylaminopropyl) carbodiimide hydrochloride salt were added into $250 \mathrm{ml}$ of $100 \mathrm{mmol} / \mathrm{L}$ phosphate buffered saline (PBS) containing $5 \mathrm{~g}$ of gelatin. The molar ratio of ethylenediamine to the carboxyl groups of gelatin was 50. The reaction mixture was agitated at $37^{\circ} \mathrm{C}$ for $18 \mathrm{~h}$ and then dialyzed against double-distilled water (DDW) for $48 \mathrm{~h}$ at room temperature. The dialyzed solution was freeze-dried to obtain a cationized gelatin. When determined using the conventional trinitrobenzene sulfonic acid method, the percentage of amino groups introduced into the gelatin was $50.9 \mathrm{~mol}$ percent per the carboxyl groups of gelatin.

\section{Preparation of Cationized Gelatin Microspheres Incorporating MMP-1 Plasmid DNA}

Cationized gelatin microspheres were prepared by chemical cross-linking of cationized gelatin in a water-in-oil emulsion state. ${ }^{13}$ Briefly, an aqueous solution of $10 \mathrm{~W} \%$ cationized gelatin $(10 \mathrm{ml})$ was preheated at $40^{\circ} \mathrm{C}$ and then added dropwise into $375 \mathrm{ml}$ of olive oil preheated to $40^{\circ} \mathrm{C}$, while an impeller stirred at $420 \mathrm{rpm}$ for $10 \mathrm{~min}$ to yield a water-in-oil emulsion. The emulsion temperature was decreased to $4^{\circ} \mathrm{C}$, followed by further stirring for $30 \mathrm{~min}$ for the natural gelation of the gelatin aqueous solution. Cold acetone $(100 \mathrm{ml})$ was added to the emulsion and stirring was continued for $10 \mathrm{~min}$. The resulting microspheres were washed 3 times with cold acetone, collected by centrifugation $\left(5,000 \mathrm{rpm}, 4^{\circ} \mathrm{C}\right.$, $5 \mathrm{~min}$ ), fractionated in size by sieves with apertures of 70 and $100 \mu \mathrm{m}$, and air-dried at $4^{\circ} \mathrm{C}$. The average diameter of the microspheres was $75 \mu \mathrm{m}$. The non-cross-linked and dried gelatin microspheres $(50 \mathrm{mg})$ were placed in $25 \mathrm{ml}$ of acetone/ $0.01 \mathrm{~mol} / \mathrm{L} \mathrm{HCl}$ solution $(7 / 3, \mathrm{vol} / \mathrm{vol})$ containing $60 \mu \mathrm{l}$ of $25 \mathrm{~W} \%$ glutaraldehyde solution and stirred at $4{ }^{\circ} \mathrm{C}$ for $24 \mathrm{~h}$ to allow the cationized gelatin to cross-link. After washing by centrifugation $\left(5,000 \mathrm{rpm}, 4^{\circ} \mathrm{C}, 5 \mathrm{~min}\right)$ with $\mathrm{DDW}$, the microspheres were agitated in $25 \mathrm{ml}$ of $100 \mathrm{mmol} / \mathrm{L}$ aqueous glycine solution at room temperature for $1 \mathrm{~h}$ to block the residual aldehyde groups of unreacted glutaraldehyde. The resulting microspheres were washed 3 times with DDW by centrifugation and freeze-dried.

Human MMP-1 genes were subcloned into mammalian transient expression vectors (pFLAG-CMV-5a; Sigma,
St Louis, MO, USA) as previously described. ${ }^{12}$ To impregnate the MMP-1 plasmid DNA into the cationized gelatin microspheres, $20 \mu \mathrm{l}$ of $100 \mathrm{mmol} / \mathrm{L}$ PBS solution (pH 7.4) containing $50 \mu \mathrm{g}$ of MMP-1 plasmid DNA was dropped onto $2 \mathrm{mg}$ of the freeze-dried cationized gelatin microspheres and left for $1 \mathrm{~h}$ to allow the plasmid DNA solution to impregnate the dried microspheres. The MMP-1 plasmid DNA was completely incorporated into the cationized gelatin microspheres by this procedure because the volume of MMP-1 plasmid DNA solution was small enough compared with that theoretically incorporated into the microspheres. A slow degradation of microspheres was expected to induce a gradual and long-lasting release of MMP-1 plasmid DNA.

\section{Animal Model and Experimental Protocol}

Male Lewis rats (10-week-old) were anesthetized with 1$1.5 \%$ isoflurane and ventilated through an endotracheal tube. MI was created by proximal ligation of the left anterior descending coronary artery (LAD) through left thoracotomy, as described previously. ${ }^{14,15}$ The rate of mortality by coronary artery ligation and the size of the MI created were similar to our previous reports. ${ }^{14,15}$ At 4 weeks following ligation, cardiac function was evaluated by echocardiography. Rats with moderate infarction (20-40\% of endocardial circumference on echocardiography) were randomized to 2 groups. Through a left thoracotomy, $100 \mu \mathrm{l}$ of PBS containing the microspheres with MMP-1 (MI+MMP-1 group, $n=14$ ) or without MMP-1 (MI group, $n=14$ ) was injected subepicardially with a fine syringe into 5 points of the periinfarct area of the LV free wall. Age- and sex-matched rats receiving neither the coronary artery ligation nor injection of PBS containing microspheres were used as controls (Normal group, $\mathrm{n=14}$ ) and were also used for the single-cell study.

\section{Evaluation of MMP-1 Plasmid DNA Release and Expression of MMP-1 In Vivo}

MMP-1 plasmid DNA and/or microspheres were radioiodinated according to the method of Chan et al. ${ }^{16}$ The 125I-labeled MMP-1 plasmid DNA was incorporated into gelatin microspheres that were later injected into rats 4 weeks after LAD ligation. To measure radioactivity, heart samples were obtained at $1,3,7,14$ and 21 days $(n=3$ at each sampling time). For the localization of MMP-1 protein, heart samples were obtained at 5 days after the injection and embedded in Tissue-Tek (Sakura Finetek USA, Torrance, CA, USA). Cryosections ( $5 \mu \mathrm{m}$ thick) were prepared from embedded samples and fixed with $4 \%$ paraformaldehyde for 20 min. Immunofluorescent examination was carried out using an M.O.M. immunodetection kit (Vector Laboratories, Burlingame, CA, USA). Each fixed section was incubated with mouse IgG blocking reagent for $1 \mathrm{~h}$. Rabbit anti-MMP1 antibody solution was diluted 100-fold with Tris buffered saline (TBS: $0.05 \mathrm{~mol} / \mathrm{L}$ Tris, $\mathrm{pH} 7.4$, with $0.15 \mathrm{~mol} / \mathrm{L} \mathrm{NaCl}$ ). These sections were incubated with the anti-rabbit IgGRITC conjugate for $1 \mathrm{~h}$ at $25^{\circ} \mathrm{C}$. Finally, the stained sections were rinsed with PBS and examined for localization of MMP-1 protein derived from the injected solution using a fluorescence microscope equipped with a digital camera. ${ }^{12}$

\section{Assessment of Cardiac Function}

Echocardiographic assessment was carried out using the method described in our previous studies. ${ }^{14,15}$ In brief, LV dimensions and function were assessed at baseline, and 2 and 4 weeks after the treatment. Images were recorded using a 10-12-MHz phased array transducer (HP SONOS 5500, 


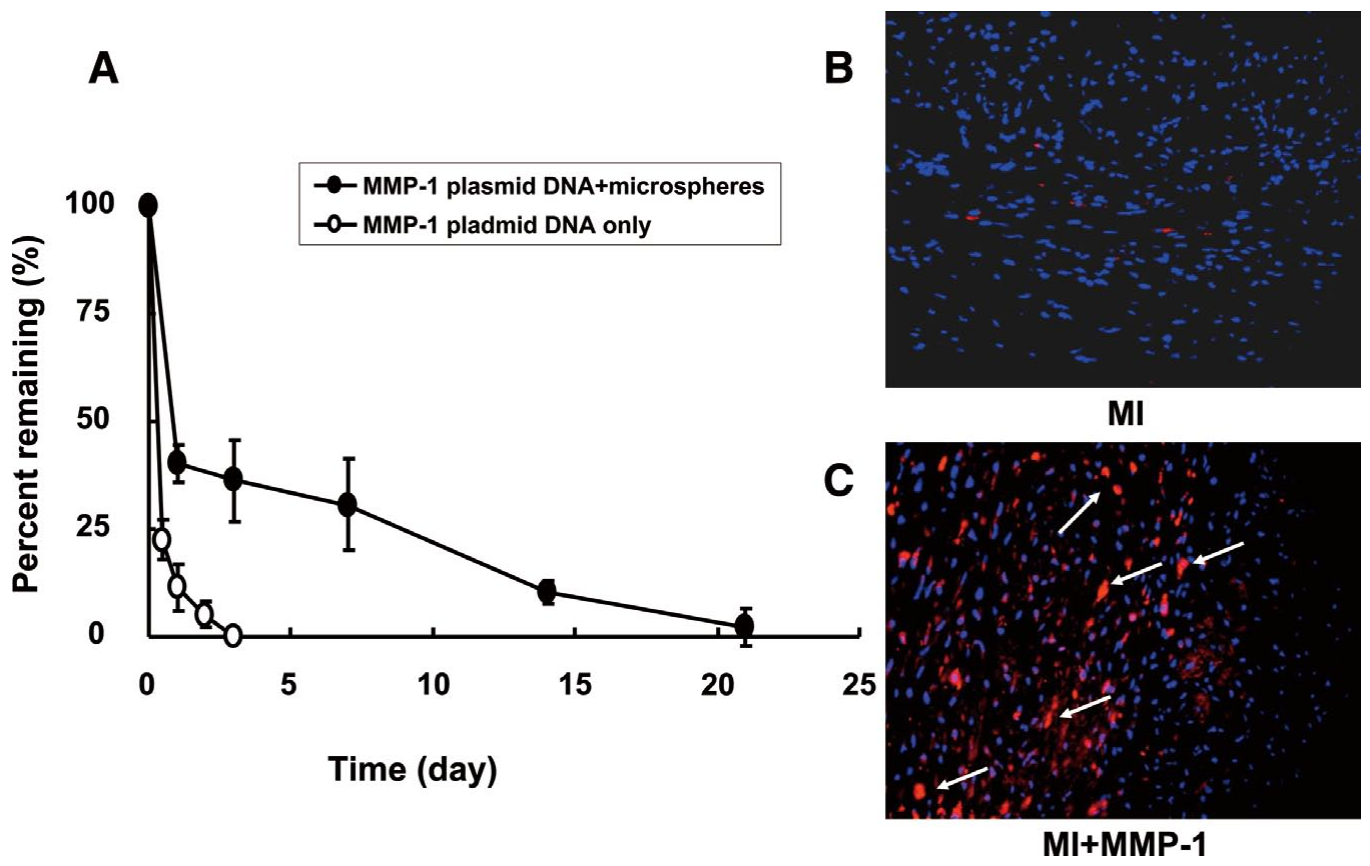

Figure 1. In vivo release of MMP-1 plasmid DNA. (A) Time profile of remaining radioactivity in the myocardium of rats after the injection of cationized gelatin microspheres incorporation ${ }^{125}$ I-labeled MMP-1 plasmid DNA. The rats were injected with the microspheres incorporating MMP-1 plasmid DNA (closed circles) and MMP-1 plasmid DNA solution (open circles). (B, C) Immunofluorescent image of MMP-1 protein visualized with RITC (red) and DAPI (blue) in the MI group (B) and MI+MMP-1 group $(\mathbf{C})$. Original magnification, $\times 400$. The MMP-1 protein secreted de novo (colored in red) was detected in rat hearts in the MI+MMP-1 group, but not detected in the MI group. Abbreviations see in text.

Andover, MA, USA). The LV end-diastolic dimension (EDD) and end-systolic dimension (ESD) were measured by M-mode tracings from the short-axis view of the LV at the papillary muscle level.14,15,17 Fractional area change (FAC) and akinetic endocardial length to the whole LV endocardial circumference (AL) were also calculated from the same short-axis view of the 2-dimensional image. All measurements were performed in a blind fashion according to the American Society for Echocardiology and averaged over 3 consecutive cardiac cycles. $14,15,17$

After the final echocardiographic examination, the rats underwent cardiac catheterization for a more precise assessment of global LV function, as described previously. ${ }^{14,15} \mathrm{In}$ brief, under general anesthesia, a $2 \mathrm{~F}$ micromanometertipped catheter (Millar Instruments Inc, Houston, TX, USA) was inserted via the right carotid artery into the LV, and a $3 \mathrm{~F}$ occlusion balloon catheter was inserted through the right femoral vein into the inferior vena cava. LV pressure and its first time-derivative $(\mathrm{dp} / \mathrm{dt})$ were continuously monitored using a multiple recording system. LV end-systolic volume was calculated using M-mode echocardiograms by the cube formula. During inferior vena cava occlusion with the balloon, pressure waveforms and $\mathrm{M}$-mode tracings were simultaneously recorded. Next, end-systolic elastance (Ees) and the time constant of isovolumic relaxation (Tau) were derived from the recorded data. In calculating Ees, the end-systolic pressure-volume points obtained from echocardiography and cardiac catheterization were subjected to least-squared linear regression. All data were acquired under stable conditions. ${ }^{14,15}$

\section{Analysis of Fibrosis}

After the final functional assessment, the rats were killed for the histological study. Masson's trichrome staining was carried out to detect fibrosis. The infarct area was divided into 5 parts, and the central (central-infarct) and lateral areas (peri-infarct) were analyzed. The percentage of fibrotic area in the non-infarct, central-infarct and peri-infarct areas was calculated in each heart using image analysis software (Scion Image Beta 4.02 Win, Scion Corporation).

\section{Electrophysiological Study of Single Myocytes}

Cardiomyocytes were isolated from the LV as previously described. ${ }^{18}$ The myocytes were superfused with a control Tyrode solution containing (in mmol/L) $140 \mathrm{NaCl}, 5.4 \mathrm{KCl}$, $1.8 \mathrm{CaCl}_{2}, 0.5 \mathrm{MgCl}_{2}, 0.3 \mathrm{NaH}_{2} \mathrm{PO}_{4}, 5.5$ glucose and 5.0 HEPES ( $\mathrm{pH}=7.4)$. Contraction was induced by applying a depolarizing pulse of $2 \mathrm{~ms}$ duration through a patch pipette using a patch clamp amplifier (Axopatch 200B, Axon Instruments), and cell length was measured every $5 \mathrm{~ms}$ with a liner image sensor (Edge Detector Hamamatsu Photonics K.K., Hamamatsu, Japan) as described previously. ${ }^{19}$ Membrane current through $\mathrm{Na}^{+}-\mathrm{Ca}^{2+}$ exchange (INCX), a pivotal protein of cardiac excitation-contraction coupling, was measured in the whole-cell clamped cardiomyocytes as extracellular $1 \mathrm{mmol} / \mathrm{L} \mathrm{Ca}^{2+}$-induced current in the same manner as in our recent study. ${ }^{18}$

\section{Northern Blot of $\mathrm{Na}^{+}-\mathrm{Ca}^{2+}$ Exchange (NCX 1)}

Northern blots, prepared with $2 \mu \mathrm{g}$ of $\operatorname{poly}(\mathrm{A})^{+}$mRNA isolated from the indicated samples, were probed in hybridization solution (Life Technologies, Inc) and 50\% formamide at $42^{\circ} \mathrm{C}$ with a radiolabeled DNA fragment derived from a coding region of the rat NCX1 (corresponding to amino acids 272-453), and washed with $0.2 \times \mathrm{SSC}$ and $0.1 \%$ SDS at $65^{\circ} \mathrm{C}$ for $1 \mathrm{~h}$. Quantification of the bands was carried out using a bioimaging analyzer (model BAS3000; Fuji Photo Film Co, Tokyo, Japan). 
A
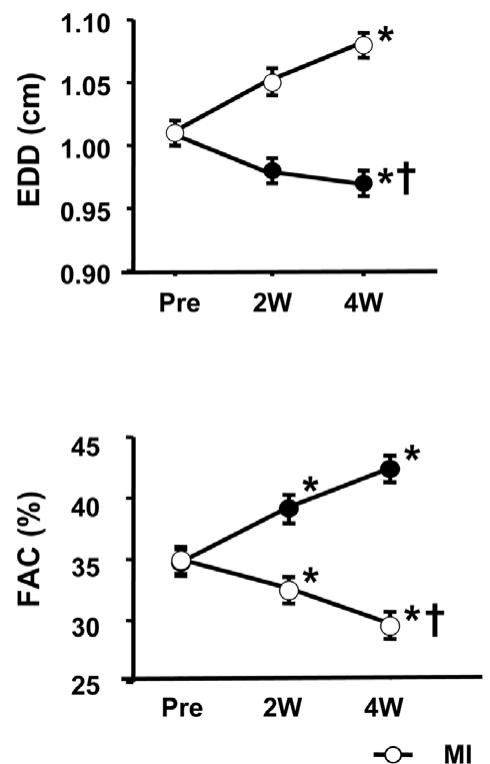
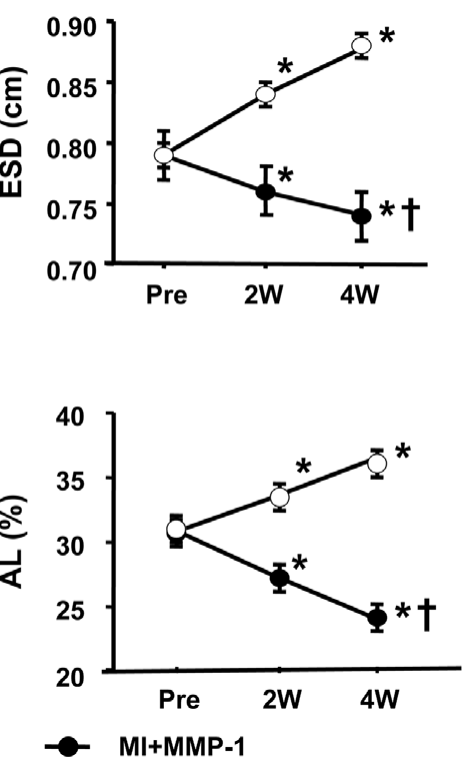
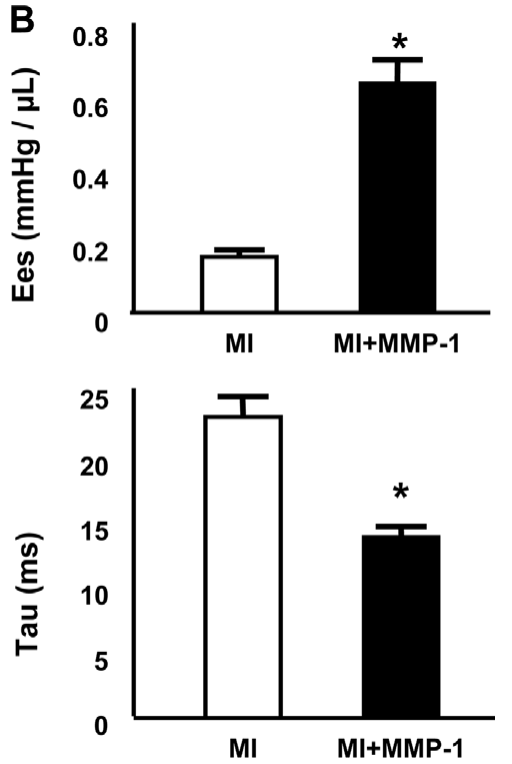

Figure 2. Assessment of cardiac function by echocardiography and catheterization. (A) Echocardiography. Data are from the MI group (open circles) and MI+MMP-1 group (closed circles). $* \mathrm{P}<0.05$ vs baseline (labeled as pre) in the same group. ${ }^{\dagger} \mathrm{P}<0.001$ vs group MI. Error bars show SEM. (B) Cardiac catheterization. ${ }^{*} \mathrm{P}<0.0001$ vs group MI. Error bars show SEM. Abbreviations see in text.

\section{Data Analysis}

All data are expressed as the mean \pm SE. Statistical analysis was carried out with ANOVA using computer software (StatView for Windows version 5.0, SAS Institute Inc, Cary, NC, USA). Multiple comparisons were carried out according to Student-Newman-Keul's method. A probability value (P) $<0.05$ was considered significant.

\section{Results}

\section{Evaluation of MMP-1 Plasmid DNA Release and Expression of MMP-1 In Vivo}

As shown in Figure 1A, ${ }^{125}$ I-labeled MMP-1 plasmid DNA was injected into the peri-infarct area of the LV free wall, and the remaining radioactivity was expressed as the percentage of radioactivity of initially injected solution containing MMP-1 plasmid DNA. MMP-1 plasmid DNA rapidly disappeared when it was injected without incorporation into the microspheres. However, MMP-1 plasmid DNA that was incorporated into the gelatin microspheres continued to exist in situ for 2-3 weeks. Furthermore, MMP-1 protein secreted de novo was detected in the MI+MMP-1 group (Figure 1C), but not in the MI group (Figure 1B), where de novo expression of MMP-1 was visualized with RITC (red).

\section{Improved Cardiac Remodeling and Function by MMP-1 Plasmid DNA}

At baseline, the heart rate was similar between the MI group and the MI+MMP-1 group ( $313 \pm 19$ vs $310 \pm 18$ beats $/ \mathrm{min}, \mathrm{P}>$ 0.05 ), as well as body weight ( $415 \pm 12$ vs $416 \pm 10 \mathrm{~g}, \mathrm{P}>0.05$ ). $\mathrm{EDD}, \mathrm{ESD}, \mathrm{FAC}$ and $\mathrm{AL}$ were also comparable between the 2 groups (Figure 2A). In the MI group, there was adverse cardiac remodeling and dysfunction, which progressively deteriorated over time, whereas in the MI+MMP-1 group there was favorable cardiac remodeling and increased wall motion in both the infarct and non-infarct remote areas. As shown in Figure 2A (Upper panel), both the EDD and ESD in the MI+MMP-1 group were significantly smaller than those in the MI group at 2 and 4 weeks after the treatment. Moreover, in the MI+MMP-1 group both the EDD and ESD decreased gradually with time after the treatment; namely, both the EDD and ESD were significantly decreased at 4 weeks than at 2 weeks $(\mathrm{P}=0.0247$ for $\mathrm{EDD}, \mathrm{P}=0.0081$ for $\mathrm{ESD})$. The longer the time after the treatment, the larger the difference between the MI and MI+MMP-1 groups for either EDD or ESD. These data suggest that the injection of MMP-1 plasmid DNA into the peri-infarct area had a beneficial effect on post-MI cardiac remodeling.

The injection of MMP-1 plasmid DNA was also able to induce improved cardiac performance, as shown by an increased percentage of kinetic area and decreased akinetic area as well as increased FAC. As shown in Figure 2A (Lower panel), the FAC in the MI+MMP-1 group was significantly higher than that in the MI group at 2 and 4 weeks after MMP-1 treatment. Moreover, in the MI+MMP-1 group FAC increased gradually with time after treatment; namely, FAC was significantly higher at 4 weeks than at 2 weeks $(\mathrm{P}=0.008)$. In the meantime, AL was significantly lower in the MI+MMP-1 group than in the MI group at 2 and 4 weeks after the treatment. Moreover, in the MI+MMP-1 group AL decreased gradually with time after treatment: AL was significantly lower at 4 weeks than at 2 weeks $(\mathrm{P}=0.0095)$. These data demonstrate that the injection of MMP-1 plasmid DNA into the peri-infarct area improved cardiac performance.

The abovementioned effects induced by exogenous MMP-1 plasmid DNA were further confirmed by cardiac catheterization. As shown in Figure 2B, Ees in the MI+ MMP-1 group was significantly higher than in the MI group $(\mathrm{P}<0.0001)$, whereas Tau in the MI+MMP-1 group was significantly shorter than in the MI group $(\mathrm{P}=0.0021)$. These data indicate that cardiac global function improved by the injection of MMP-1 plasmid DNA. 


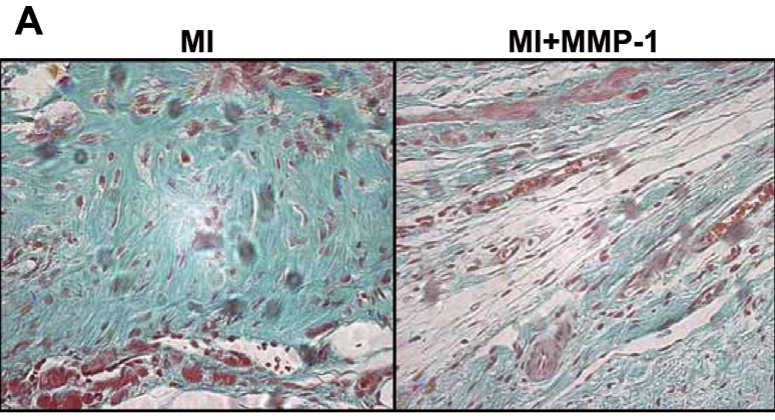

B

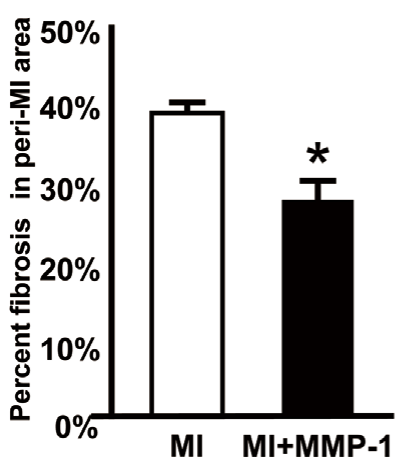

C

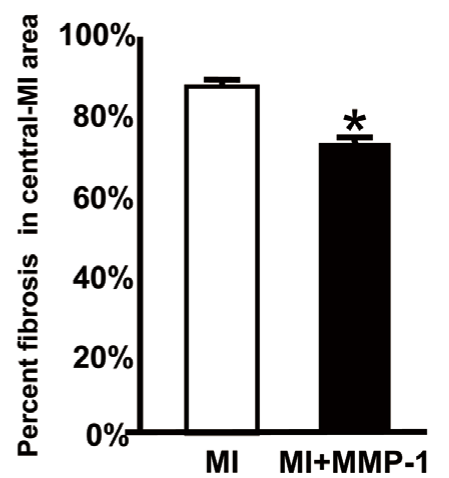

MI

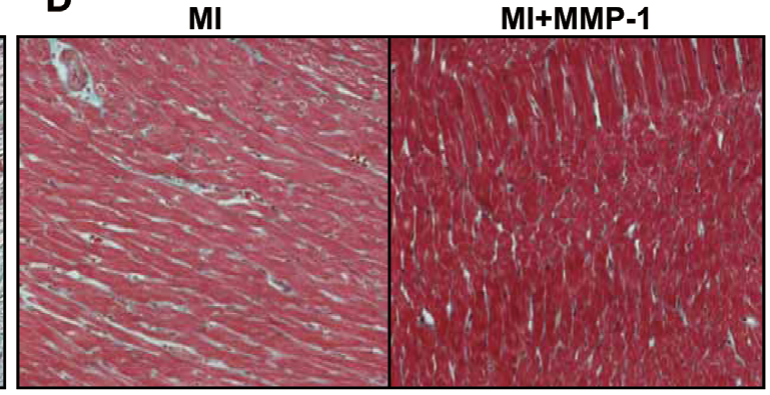

E

Figure 3. Assessment of myocardial fibrosis by immunohistochemistry with Masson's trichrome staining. (A) Fibrosis stained blue by Masson's trichrome in the peri-infarct area. Original magnification, $\times 400$. (B) Fibrosis as a percentage in the peri-infarct area $(\mathrm{n}=10)$. $* \mathrm{P}=0.0003$ vs MI group. $(\mathbf{C})$ Fibrosis as a percentage in the central-infarct area $(\mathrm{n}=10)$. $* \mathrm{P}<$ 0.0001 vs MI group. (D) Fibrosis stained blue by Masson's trichrome in the non-infarct area. Original magnification, $\times 400$.

(E) Fibrosis as a percentage in the non-infarct area $(\mathrm{n}=10)$. ${ }^{\dagger} \mathrm{P}=0.0007$ vs MI group. Abbreviations see in text.

Decreased Myocardial Fibrosis by MMP-1 Plasmid DNA

The fibrotic area evaluated with Masson's trichrome staining was significantly decreased in the MI+MMP-1 group compared with the MI group in both the peri-infarct $(\mathrm{P}=$ 0.0003 , Figures 3A, B) and central-infarct areas $(P<0.0001$, Figure 3C). The decrease was also found in the non-infarct regions $(\mathrm{P}=0.0007$, Figures 3D, E) in which the percentage of fibrosis was comparable to that of the normal heart. The decrease in fibrotic area might be an explantaion for the improved cardiac function.

Increased Myocyte Shortening by MMP-1 Plasmid DNA To evaluate cardiomyocyte contractility, cardiomyocytes were isolated from the non-infarct regions of the LV free wall. The degree of cell shortening (\% shortening), which was initiated by electrical stimuli, was assessed by monitoring the longitudinal cell length (Figure 4: summary of the pooled data at different beating frequencies from the Normal, MI and MI+MMP-1 groups). Cell shortening from the MI group was depressed when compared with that from the Normal group ( $\mathrm{P}=0.0389)$. The MMP-1 treatment augmented the \% cell shortening to almost the same degree as in the Normal group at $0.1 \mathrm{~Hz}, 1 \mathrm{~Hz}$ and $2.5 \mathrm{~Hz}$, respectively.

\section{Reduced $\mathrm{Na}^{+}-\mathrm{Ca}^{2+}$ Exchange Current (INCX) by MMP-1 Plasmid DNA}

Up-regulation of $\mathrm{Na}^{+}-\mathrm{Ca}^{2+}$ exchange function has been recognized a characteristic biomarker in post-MI heart failure. ${ }^{20}$ As shown in Figure 5, the expression of NCX1 protein and membrane current through the NCX1 (INCX) were enhanced in the MI group. In the MI+MMP-1 group, INCX was significantly reduced to a similar level as in the

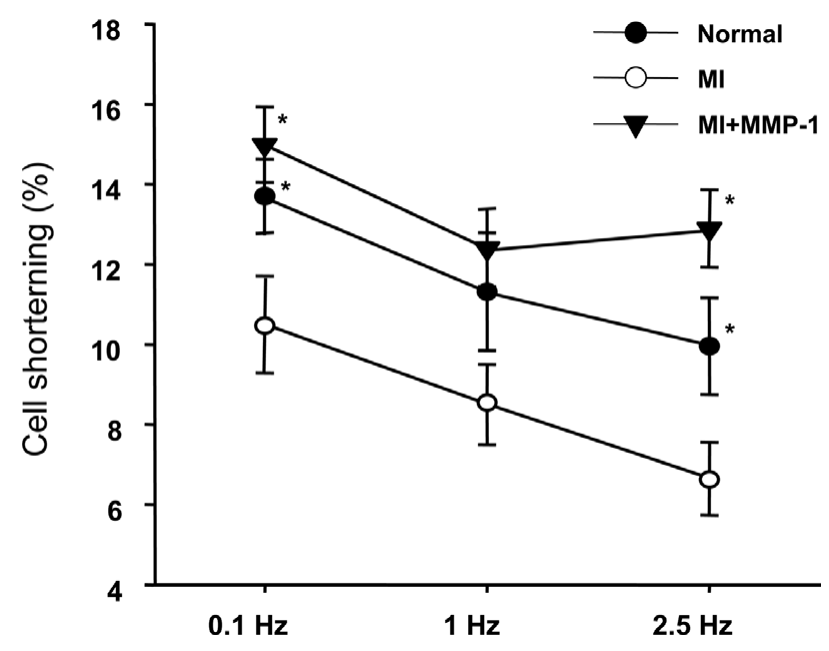

Figure 4. Contractility of isolated cardiomyocyte. Comparison of cardiomyocyte contractility among Normal, MI and MI+MMP-1 groups. Contractility was expresses as a \% shortening of cell length. Single cell contractility markedly improved in the MI+MMP-1 group over a wide range of beating rates. $* \mathrm{P}<0.05$ vs MI group. Abbreviations see in text.

Normal group, but no significant decrease in mRNA encoding NCX1 was found.

\section{Discussion}

In this study, we used a new technique of cationizing gelatin microsphere incorporating MMP-1 plasmid DNA to express MMP-1 protein locally in the peri-MI area of chronic MI rat 
A

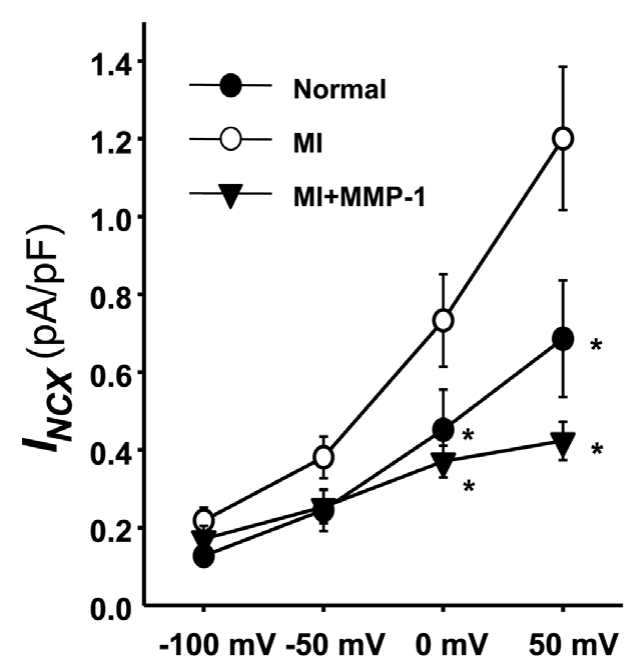

B

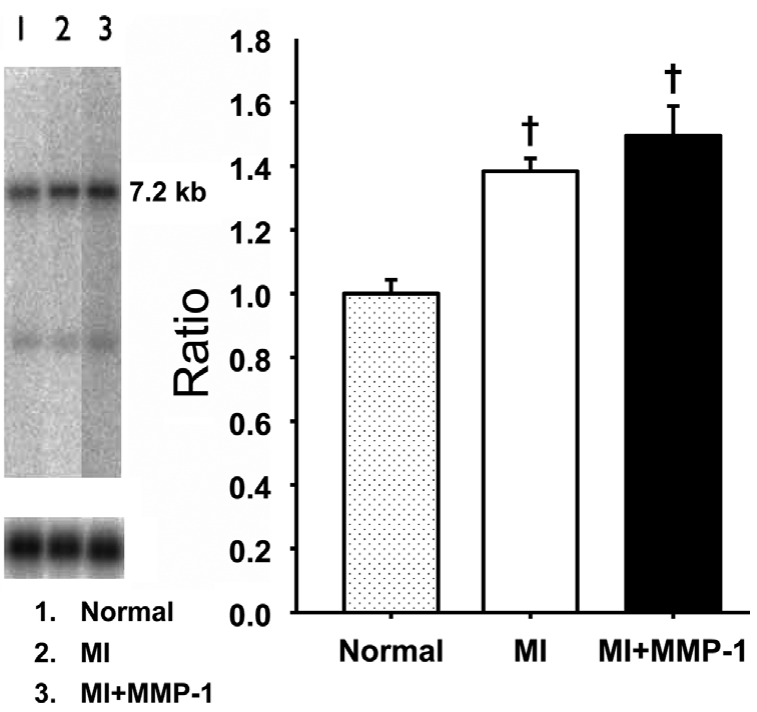

Figure 5. $\mathrm{Na}^{+}-\mathrm{Ca}^{2+}$ exchange function. (A) INCX. INCX was normalized to cell capacitance and plotted at different membrane potentials. (B) Northern blot analysis of NCX1 mRNA from rat hearts. Each lane contains $2 \mu \mathrm{g}$ poly (A)+ RNA extracted from the indicated sample. The size of the mRNA for NCX1 was estimated to be 7.2 kilobases (Left upper). Glyceraldehyde-3-phosohate dehydrogenase (Left lower) was used as a control. (Right) Quantification of band intensity obtained by northern blot. Bar diagram showing relative expression levels (mean \pm SEM) of NCX1 in normals $(n=4)$, MI $(\mathrm{n}=4)$ and MI+MMP-1 $(\mathrm{n}=4)$ rat ventricles. ${ }^{\dagger} \mathrm{P}<0.01$ vs normal group. Abbreviations see in text.

hearts for a limited period of time. The release of MMP-1 plasmid DNA peaked within $24 \mathrm{~h}$ after injection and continued to be released for 3 weeks. The released MMP-1 plasmid DNA directly permeated the myocardium in situ and resulted in the production of MMP-1 protein. The exogenously expressed MMP-1 then degraded collagen and attenuated myocardial fibrosis, thus improving myocyte contractility and leading to recovery of overall cardiac function. Our study demonstrated that inhibition of excessive accumulation of collagen could be a beneficial therapeutic strategy for chronic MI heart failure. Although MMP-1 does not express in rat hearts, ${ }^{21,22}$ it possesses a high substrate specificity for fibrillar collagen, ${ }^{8}$ which could be advantageous from a therapeutic viewpoint. However, its use as a therapeutic approach has been limited by its short bioavailability. Our method using cationized gelatin microspheres overcomes this shortcoming and enables MMP-1 to express its biological activity for an appropriate time when collagen fibrosis increases in heart tissue.

It has been demonstrated that after the development of a transmural MI in rats, adverse ventricular remodeling by fibrous tissue occurs at both the site of MI and in remote myocardium. ${ }^{23-26}$ Although the increase in reparative fibrosis and ECM serves to preserve the structural integrity of infarcted tissue in the early phase of MI, in the chronic phase, the accumulation of a large amount of fibrosis, especially interstitial fibrosis, leads to ventricular diastolic dysfunction rather than preservation of cardiac structure. ${ }^{27-30}$ In our rat model of MI, heart function continued to deteriorate 4-8 weeks after MI in the MI group, while the injection of MMP-1 plasmid DNA significantly improved heart function. These results imply that cardiac fibrosis continued to progress for 8 weeks after MI in our rat model. Although the detailed time course of fibrosis remains to be clarified, our study demonstrates that inhibition of excessive accumulation of collagen was indeed beneficial in this animal model. As demonstrated in Figure 3, fibrosis was improved in the central and non-MI areas, as well as the peri-MI region where the MMP-1 plasmid was injected. Regarding the central MI area, it is likely that the released MMP-1 plasmid DNA diffused and was expressed in the central MI area because of the small size of the rat heart. The improved contractility of the peri-MI area might indirectly improve both the fibrosis and contractility of the nonMI areas that are connected with each other. The mechanism underlying this remote effect remains to be studied.

The underlying mechanism for the beneficial effects of controlled release of MMP-1 plasmid DNA needs to be further studied. The improvement in wall motion in the infarct area might be a result of degradation of interstitial collagen, which might decrease the stiffness of the ventricle. The increase in contractility of the remaining myocytes is likely to be 1 of the causes of improved systolic function. The cause-and-effect relationship of increased contractility of single cardiomyocytes is not totally clear in this study. However, because the function of NCX1, an important protein for cardiac $\mathrm{Ca}^{2+}$ handling, returned to normal levels after the MMP-1 plasmid treatment, an alteration in intracellular $\mathrm{Ca}^{2+}$ handling might be associated with this effect. The alteration might be caused indirectly by a decrease in the stiffness of the ventricle or by degradation of intracellular proteins by MMP-1, as demonstrated with MMP-2.31

Previous studies have demonstrated that overexpression of human MMP-1 within the normal myocardium of mice results in significant myocardial collagen disruption and LV dysfunction at 12 months of age, ${ }^{32}$ whereas it prevents LV dilatation and preserves cardiac function in a mouse model of pressure overload. ${ }^{11}$ Therefore, it is speculated that induction of MMP-1 is beneficial under pathophysiological conditions in which collagen accumulates in the heart, such as chronic MI (as in this study) and sustained hypertension. ${ }^{11}$ Conversely, upregulation of MMP-1 in the normal heart may result in deterioration of cardiac function. 


\section{Study Limitations}

First, the dose-dependent effect of MMP-1 needs to be further studied. We speculate that the expression level of MMP-1 is a key issue preventing MMP-1 from providing beneficial effects. A level of exogenous MMP-1 that is too high may even be detrimental to cardiac remodeling, leading to cardiac rupture. A level of MMP-1 that is too low may be inadequate to exert beneficial effects. The time-dependent response after the onset of infarction is also an important issue, not only in terms of efficacy but also safety. Second, further studies are needed to elucidate the underlying mechanisms by which the MMP-1 plasmid DNA microspheres improve cardiac function and the causative relationship between plasmid delivery and the observed effects. There is increasing evidence to suggest that alterations in the MMP/tissue inhibitor of MMP (TIMP) balance may contribute to myocardial fibrosis. ${ }^{8-11,13,21,22,24,31,32}$ Therefore, the induction of exogenous MMP-1 might affect other MMPs and TIMP, which are normally expressed in the heart. It is also possible that MMP-1 acts on non-ECM proteins both inside and outside the cell. ${ }^{31}$ Nevertheless, to the best of our knowledge the present study provides evidence for the first time that the local delivery of exogenous MMP-1 plasmid DNA with microspheres significantly improves cardiac remodeling and cardiac function.

\section{Conclusion}

The present study convincingly demonstrated that controlreleased administration of exogenous MMP-1 plasmid DNA is indeed able to effectively prevent the progression of adverse cardiac remodeling in a chronic MI heart failure model in rats.

\section{Acknowledgments}

The authors thank Mrs Fumiyo Kataoka for her meticulous work on the histological studies. This study was supported by grants from the Ministry of Education, Culture, Sports, Science and Technology Japan (grant numbers 13470271 to M.K. and 17590186 to S.M.), and a Grant-in-Aid for Scientific Research (KAKENHI), Kyoto, Japan (grant number 19P07085 to X.L.), Japan Society for Promotion of Science (JSPS).

\section{References}

1. Pfeffer MA, Braunwald E. Ventricular remodeling after myocardial infarction: Experimental observations and clinical implications. Circulation 1990; 81: $1161-1172$.

2. Reimer KA, Vander Heide RS, Richard VJ. Reperfusion in acute myocardial infarction: Effect of timing and modulating factors in experimental models. Am J Cardiol 1993; 72: 13G-21G.

3. Hosenpud JD, Bennett LE, Keck BM, Boucek MM, Novick RJ. The registry of the international society for heart and lung transplantation: Seventeenth official report-2000. J Heart Lung Transplant 2000; 19: 909-931.

4. Allori AC, Sailon AM, Warren SM. Biological basis of bone formation, remodeling, and repair-part II: Extracellular matrix. Tissue Eng Part B Rev 2008; 14: 275-283.

5. Jezierska A, Motyl T. Matrix metalloproteinase-2 involvement in breast cancer progression: A mini-review. Med Sci Monit 2009; 15: RA32-RA40.

6. Fujimoto S, Hartung D, Ohshima S, Edwards DS, Zhou J, Yalamanchili $\mathrm{P}$, et al. Molecular imaging of matrix metalloproteinase in atherosclerotic lesions: Resolution with dietary modification and statin therapy. J Am Coll Cardiol 2008; 52: 1847-1857.

7. West-Mays JA, Pino G. Matrix metalloproteinases as mediators of primary and secondary cataracts. Expert Rev Ophthalmol 2007; 2: 931-938.

8. Spinale FG. Myocardial matrix remodeling and the matrix metalloproteinases: Influence on cardiac form and function. Physiol Rev 2007; 87: $1285-1342$.

9. Spinale FG, Coker ML, Bond BR, Zellner JL. Myocardial matrix degradation and metalloproteinase activation in the failing heart: A potential therapeutic target. Cardiovasc Res 2000; 46: 225-238.

10. Parsons SL, Watson SA, Brown PD, Collins HM, Steele RJ. Matrix metalloproteinases. Br J Surg 1997; 84: 160-166.

11. Foronjy RF, Sun J, Lemaitre V, D'Armiento JM. Transgenic expression of matrix metalloproteinase-1 inhibits myocardial fibrosis and prevents the transition to heart failure in a pressure overload mouse model. Hypertens Res 2008; 31: 725-735.

12. Aoyama T, Yamamoto S, Kanematsu A, Ogawa O, Tabata Y. Local delivery of matrix metalloproteinase gene prevents the onset of renal sclerosis in streptozotocin-induced diabetic mice. Tissue Eng 2003; 9: $1289-1299$.

13. Fukunaka Y, Iwanaga K, Morimoto K, Kakemi M, Tabata Y. Controlled release of plasmid DNA from cationized gelatin hydrogels based on hydrogel degradation. J Control Release 2001; 80: $333-$ 343.

14. Lin X, Fujita M, Kanemitsu N, Kimura Y, Tambara K, Premaratne GU, et al. Sustained-release erythropoietin ameliorates cardiac function in infracted rat-heart without inducing polycythemia. Circ J 2007; 71: 132-137.

15. Tambara K, Premaratne GU, Sakaguchi G, Kanemitsu N, Lin X, Nakajima $\mathrm{H}$, et al. Administration of control-released hepatocyte growth factor enhances the efficacy of skeletal myoblast transplantation in rat infarcted hearts by greatly increasing both quantity and quality of the graft. Circulation 2005; 112: I-129-I-134.

16. Chan HC, Ruyechan WT, Wetmur JG. In vitro iodination of low complexity nucleic acids without chain scission. Biochemistry 1976; 15: $5487-5490$.

17. Litwin SE, Katz SE, Morgan JP, Douglas PS. Serial echocardiographic assessment of left ventricular geometry and function after large myocardial infarction in the rat. Circulation 1994; 89: 345-354.

18. Lin X, Jo H, Sakakibara Y, Tambara K, Kim B, Komeda M, et al. Beta-adrenergic stimulation does not activate $\mathrm{Na}+\mathrm{Ca} 2+$ exchange current in guinea pig, mouse, and rat ventricular myocytes. Am J Physiol Cell Physiol 2006; 290: C601-C608.

19. Jo H, Noma A, Matsuoka S. Calcium-mediated coupling between mitochondrial substrate dehydrogenation and cardiac workload in single guinea-pig ventricular myocytes. J Mol Cell Cardiol 2006; 40: $394-404$.

20. Xiong W, Tian Y, DiSilvestre D, Tomaselli GF. Transmural heterogeneity of $\mathrm{Na}^{+}-\mathrm{Ca}^{2+}$ exchange: Evidence for differential expression in normal and failing hearts. Circ Res 2005; 97: 207-209.

21. Li H, Simon H, Bocan TM, Peterson JT. MMP/TIMP expression in spontaneously hypertensive heart failure rats: The effect of ACE- and MMP-inhibition. Cardiovasc Res 2000; 46: 298-306.

22. Peterson JT, Li H, Dillon L, Bryant JW. Evolution of matrix metalloprotease and tissue inhibitor expression during heart failure progression in the infarcted rat. Cardiovasc Res 2000; 46: 307-315.

23. Sun Y, Zhang JQ, Zhang JK, Lamparter S. Cardiac remodeling by fibrous tissue after infarction in rats. J Lab Clin Med 2000; 135: $316-323$.

24. Etoh T, Joffs C, Deschamps AM, Davis J, Dowdv K, Hendrick J. Myocardial and interstitial matrix metalloproteinase activity after acute myocardial infarction in pigs. Am J Physiol Heart Circ Physiol 2001; 281: H987-H994.

25. Spinale FG. Matrix metalloproteinases: Regulation and dysregulation in the failing heart. Circ Res 2002; 90: 520-530.

26. Masano T, Kawashima S, Toh R, Satomi-Kobayashi S, Shinohara M, Takaya $\mathrm{T}$, et al. Beneficial effects of exogenous tetrahydrobiopterin on left ventricular remodeling after myocardial infarction in rats: The possible role of oxidative stress caused by uncoupled endothelial nitric oxide synthase. Circ J 2008; 72: 1512-1519.

27. Weber KT. Cardiac interstitium in health and disease: The fibrillar collagen network. J Am Coll Cardiol 1989; 13: 1637-1652.

28. Anversa P, Li P, Zhang X, Olivetti G, Capasso JM. Ischemic myocardial injury and ventricular remodeling. Cardiovasc Res 1993; 27: $145-157$.

29. Swan HJC. Left ventricylar dysfunction in ischemic heart disease: Fundamental importance of fibrous matrix. Cardiovasc Drugs Ther 1994; 8: $305-312$.

30. Dixon IM, Ju H, Jassal DS, Peterson DJ. Effect of ramipril and losartan on collagen expression in right and left heart after myocardial infarction. Mol Cell Biochem 1996; 165: 31-45.

31. Schulz R. Intracellular targets of matrix metalloproteinase-2 in cardiac disease: Rationale and therapeutic approaches. Аппи Rev Pharmacol Toxicol 2007; 47: 211-242.

32. Kim HE, Dalal SS, Young E, Legato MJ, Weisfeldt ML, D'Armiento J. Disruption of the myocardial extracellular matrix leads to cardiac dysfunction. J Clin Invest 2000; 106: 857-866. 